\title{
EL PAPEL DE LOS PLANES DE IGUALDAD EN LA IMPLANTACIÓN DE MEDIDAS DE IGUALDAD EFECTIVA EN LAS EMPRESAS
}

\author{
Equal Plans and Real Equal Mesaures at Entreprises
}

\author{
ELISA Sierra Hernáiz \\ Titular de Derecho del Trabajo y de la Seguridad Social \\ Universidad Pública de Navarra
}

Orcid: 0000-0002-4320-026X

Recibido: 30.10 .2017

DOI: $10.1387 /$ lan-harremanak. 18857

Aceptado: 20.11.2017

\section{ABSTRACT}

En el presente trabajo se estudia el papel desempeñado por los Planes de igualdad como instrumentos del principio de igualdad sustancial para superar los efectos de la brecha de género, que se manifiesta en todos los ámbitos de la relación laboral $y$ que tanto dificulta la verdadera igualdad de oportunidades entre trabajadores $y$ trabajadoras. Para ello, se analiza su contenido con una perspectiva critica, teniendo en cuenta que ya han pasado diez años desde la aprobación de la Ley Orgánica de Igualdad, para, a posteriori, proponer posibles medidas de actuación para que los Planes de igualdad sean un instrumento eficaz para la consecución de los objetivos previstos en dicha norma y no se conviertan en meras declaraciones de principios o establezcan medidas no conectadas con la realidad para la que están pensados.

Palabras clave: Planes de igualdad, igualdad sustancial, brecha de género.

This paper analyzes the role of Equality Plans as an instruments of the principle of real equality to overcome the effects of the gender gap in all areas of the employment relationship that hinder true equality of opportunities between workers. To 
achieve this goal, its content is studied with a critical perspective, taking into account that ten years have passed since the Law was passed. Its allows proposing new measures so that the Equality Plans are an effective instrument for the achievement of the law's objectives and don not become statements of principles or establish measures not connected with the real situation of women at work.

Keywords: Equality Plans, real equality, gender gap. 


\section{SUMARIO}

1. Introducción. 2. Referencias a la noción de igualdad substancial en la ley orgánica para de igualdad efectiva entre hombres y mujeres y su impacto en el estatuto de los trabajadores frente al principio general de igualdad formal o igualdad de trato. 3. La situación actual de la mujer en el mercado laboral con respecto a la brecha de género. 4 . El papel de la negociación colectiva en general y de los planes de igualdad en particular en la consecución del principio de igualdad sustancial. 4.1. Delimitación de las funciones en materia de igualdad. 4.2. Análisis de planes de igualdad y convenios colectivos desde la perspectiva del principio de igualdad sustancial. 5. Propuestas de actuación para los planes de igualdad desde la perspectiva del principio de igualdad sustancial. 6. A modo de conclusión. 7. Bibliografía.

\section{Introducción}

Este año se han cumplido diez años de la aprobación de la Ley Orgánica 3/2007, de 22 de marzo, para la igualdad efectiva de mujeres y hombres (LOI), y si bien no se pueden negar los logros obtenidos, hoy en día persiste una desigualdad estructural de género, tanto a nivel social como en el ámbito específico de las relaciones laborales, cuya principal manifestación es la brecha de género en el mercado laboral español.

Una de las principales novedades de la Ley fue la regulación de los planes de igualdad, asignándoles un rol fundamental para el logro de la igualdad real, y no meramente formal, mediante actuaciones concretas y específicas en cada empresa o centro de trabajo que tengan en cuenta la realidad de sus condiciones laborales $^{1}$, más allá de declaraciones genéricas de principios e intenciones que tan pocos avances han tenido hasta el momento en el ámbito de la negociación colectiva, actuaciones que tienen cabida en el contenido de los Planes de igualdad dada la amplitud de materias y la diversidad de medidas que pueden establecer ${ }^{2}$, que abarcan, a modo de ejemplo, desde acabar con las situaciones de discrimina-

\footnotetext{
1 Respecto a la aplicación de medidas de igualdad sustancial en el ámbito de la seguridad social, véase: Vicente (2012: 192 y ss.).

2 En general, para un análisis del contenido de las medidas de los planes de igualdad y su papel en esta materia véase: Nieto (2015: 160 y ss.); Martínez (2015: 414 y ss.) y Olarte (2016: 535 y ss.).
} 
ción indirecta — la desigualdad salarial es una manifestación de ella—o establecer medidas de acción positiva — más radicales - para la promoción y ascenso de las trabajadoras dentro la empresa. Lógicamente, la efectividad de los Planes de igualdad va a depender, en gran medida, de los incentivos de la legislación, que se convertirán en un referente a la hora de idear e implantar las concretas actuaciones ${ }^{3}$.

3 En este sentido, cabe citar la Disposición transitoria tercera. Políticas activas de empleo dirigidas a mujeres del Real Decreto Legislativo 3/2015, de 23 de octubre, por el que se aprueba el texto refundido de la Ley de Empleo, BOE, de 24 de octubre de 2015, que establece:

"En tanto subsistan las actuales tasas de ocupación y de paro respecto de la población activa femenina, los poderes públicos deberán organizar la gestión de las politicas activas de empleo de tal forma que el colectivo femenino se beneficie de la aplicación de tales politicas en una proporción equivalente a su peso en el colectivo de los desempleados".

Igualmente, el III Acuerdo para el empleo y la negociación colectiva 2015-2017, BOE, de 20 de junio de 2015, señala los criterios de igualdad de género, como son:

"Incluir criterios en materia de igualdad de oportunidades entre hombres y mujeres, a tener en cuenta en la negociación colectiva, sobre: incorporación de cláusulas de acción positiva; establecimiento de sistemas de selección, clasificación, promoción y formación; eliminación de denominaciones sexistas en la clasificación profesional; subsanación de diferencias retributivas. * Incorporar medidas o criterios que mejoren el acceso al empleo, la promoción y la formación, prestando especial atención a la contratación de mujeres en aquellos sectores en los que se encuentren subrepresentadas. ${ }^{*}$ Trasladar aquellas materias que el Estatuto de los Trabajadores o la Ley Orgánica para la igualdad efectiva de mujeres y hombres reenvían a la negociación colectiva o al acuerdo con el empresario. ${ }^{*}$ Abordar en el ámbito sectorial criterios relativos a los contenidos de los Planes de igualdad referidos en sus ámbitos de aplicación, evaluación, seguimiento, administración y resolución de las discrepancias. ${ }^{*}$ Establecer medidas de conciliación de la vida laboral y personallfamiliar que compatibilicen las necesidades de los trabajadores y de las empresas. * Abordar la prevención del acoso sexual y el acoso por razón de sexo, a través de medidas de prevención como la negociación de protocolos de actuación que permitan erradicar este tipo de acciones y actitudes en las empresas. * Facilitar a través de la negociación colectiva el ejercicio efectivo de los derechos reconocidos en el ámbito laboral a las víctimas de violencia de género por la Ley Orgánica 1/2004, de 28 de diciembre, de medidas de protección integral contra la violencia de género».

A su vez, el Eje 4. Igualdad de oportunidades en el acceso al empleo del Real Decreto 751/2014, de 5 de septiembre, por el que se aprueba la Estrategia Espańola de Activación para el empleo 20142016, BOE, de 23 de septiembre de 2014, indica como objetivos estructurales:

«4.1 Remover obstáculos en el acceso al empleo: Establecer medidas que eliminen o disminuyan los obstáculos relacionados con el lugar de residencia, la situación socioeconómica o determinadas circunstancias personales, especialmente en aquellos sectores en los que las mujeres se encuentran subrepresentadas. 4.2 Promover la movilidad funcional y sectorial: Promover la movilidad funcional y sectorial, especialmente en aquellos sectores o cualificaciones con baja oferta en el mercado laboral actual y en el entorno de la propia empresa. 4.3 Promover la movilidad geográfica: Promover la movilidad geográfica para aprovechar oportunidades de empleo y formación que existan en otras zonas del país y/o el extranjero. 4.4 Promover medidas de promoción de la conciliación de la vida familiar y laboral y la corresponsabilidad» o el Compromiso estratégico para la igualdad de género 2016-2019, adoptado por la Comisión Europea - Strategic Engagement for Gender Equality, 2016-2019, Luxemburg 2015 (http:// ec.europa.eu/ justice/gender-equality/document/index; https://www.mecd.gob.es/ministerio-mecd/organizacion/ espacio-de-igualdad/planes-estrategicos.html) — que sitúa entre sus áreas prioritarias: «aumentar la participación de las mujeres en el mercado laboral y promover la igualdad en la independencia económica de mujeres y hombres», así como «reducir las disparidades entre sexos en las retribuciones, los ingresos y las 
Por todo ello, teniendo en cuenta el tiempo transcurrido y la variedad de Planes de igualdad puestos en marcha, es posible realizar un estudio para determinar si hasta el momento han sido o no eficaces en la consecución de los objetivos previstos por la LOI desde la perspectiva de la igualdad material o real.

\section{Referencias a la noción de igualdad substancial en la Ley Orgánica para de Igualdad efectiva entre hombres y mujeres y su impacto en el Estatuto de los Trabajadores frente al principio general de igualdad formal o igualdad de trato}

A lo largo del texto de la LOI es posible encontrar continuas referencias al principio de igualdad sustancial ${ }^{4}$. Así, además de la propia denominación de la Ley, en el preámbulo se hace mención a los artículos 14 y 9.2 de la Constitución Española (CE) para resaltar que

Resulta necesaria, en efecto, una acción normativa dirigida a combatir todas las manifestaciones aún subsistentes de discriminación, directa o indirecta, por razón de sexo y a promover la igualdad real entre mujeres y hombres, con remoción de los obstáculos y estereotipos sociales que impiden alcanzarla.

Merece, asimismo, destacarse que la Ley prevea, con el fin de alcanzar esa igualdad real efectiva entre mujeres y hombres, un marco general para la adopción de las llamadas acciones positivas. Se dirige, en este sentido, a todos los poderes públicos un mandato de remoción de situaciones de constatable desigualdad fáctica, no corregibles por la sola formulación del principio de igualdad jurídica o formal. Y en cuanto estas acciones puedan entrañar la formulación de un derecho desigual en favor de las

\footnotetext{
pensiones, $y$ de esa manera combatir la pobreza entre las mujeres» mediante acciones para " ${ }^{*}$ Alcanzar el objetivo europeo del 75 por 100 de mujeres y hombres en el empleo en 2020 y cerrar la brecha de empleo de las mujeres. * Repartir más igualitariamente entre mujeres y hombres el tiempo dedicado a las responsabilidades de cuidados y en el hogar, y mejorar las posibilidades de conciliar las responsabilidades profesionales y de cuidados. * Alcanzar los objetivos del Consejo Europeo de Barcelona sobre cuidado de menores, $y$ considerar una seria reflexión con los Estados miembros sobre posibles vías para hacerlos más ambiciosos, asi como extenderlos a la cobertura del cuidado de otros dependientes. ${ }^{*}$ Promover el emprendimiento por las mujeres y la igualdad de género en la investigación. * Integrar mejor en el mercado de trabajo a las mujeres migrantes».

${ }^{4}$ La Ley Orgánica de Igualdad en gran medida es deudora del derecho comunitario cuya principal norma de referencia es la Directiva 2006/54, de 5 de julio de 2006, relativa a la aplicación del principio de igualdad de oportunidades entre hombres y mujeres en asuntos de empleo y ocupación (refundición) que postula por una prevalencia del principio de igualdad formal o de trato — «Artículo 1. Finalidad. La presente Directiva tiene por objeto garantizar la aplicación del principio de igualdad de oportunidades e igualdad de trato entre hombres y mujeres en asuntos de empleo y ocupación»- integrando puntualmente principios de igualdad material a lo largo de su regulación, como son las medidas de acción positiva - "Artículo 3. Acción positiva. Los Estados miembros podrán mantener o adoptar las medidas indicadas en el artículo 141, apartado 4, del Tratado con objeto de garantizar en la práctica la plena igualdad entre hombres y mujeres en la vida laborali.
} 
mujeres, se establecen cautelas y condicionamientos para asegurar su licitud constitucional.

De esta primera aproximación al texto legal se extrae la necesidad de ir más allá del principio de igualdad formal o de trato si realmente se quiera acabar con la situación de discriminación y desigualdad de la mujer en la sociedad. Sin embargo, la concreta regulación de la LOI apuesta, como principio general de actuación, por el principio de igualdad formal. Así, en el artículo 1 se señala que su objeto es: "hacer efectivo el derecho de igualdad de trato y de oportunidades entre mujeres...”. A su vez, como muestra clara de que dicha norma apuesta por la aplicación de este principio, cabe citar el artículo 2, que garantiza que, dentro de su ámbito de aplicación "Todas las personas gozarán de los derechos derivados del principio de igualdad de trato y de la prohibición de discriminación por razón de sexo»; el artículo 3: "El principio de igualdad de trato entre mujeres y hombres supone la ausencia de toda discriminación, directa o indirecta, por razón de sexo, y, especialmente, las derivadas de la maternidad, la asunción de obligaciones familiares y el estado civil» y los artículos 5 - Igualdad de trato y de oportunidades en el acceso al empleo, en la formación y en la promoción profesionales, y en las condiciones de trabajo- y 6 - Conceptos de discriminación directa e indirecta-.

Frente a este carácter generalista, es posible encontrar a lo largo de la norma manifestaciones del principio de igualdad sustancial. En concreto, el artículo 11 reconoce la legitimidad constitucional y legal de las medidas de acción positiva

1. Con el fin de hacer efectivo el derecho constitucional de la igualdad, los Poderes Públicos adoptarán medidas específicas en favor de las mujeres para corregir situaciones patentes de desigualdad de hecho respecto de los hombres. Tales medidas, que serán aplicables en tanto subsistan dichas situaciones, habrán de ser razonables y proporcionadas en relación con el objetivo perseguido en cada caso. 2. También las personas físicas y jurídicas privadas podrán adoptar este tipo de medidas en los términos establecidos en la presente Ley.

Por lo que se refiere al ámbito de las relaciones laborales, regulado en el Título IV. El derecho al trabajo en igualdad de oportunidades, establece, en el artículo 42, que los programas de mejora de la empleabilidad de las mujeres puedan tener como objetivo prioritario de

aumentar la participación de las mujeres en el mercado de trabajo y avanzar en la igualdad efectiva entre mujeres y hombres. Para ello, se mejorará la empleabilidad y la permanencia en el empleo de las mujeres, potenciando su nivel formativo y su adaptabilidad a los requerimientos del mercado de trabajo. 2. Los Programas de inserción laboral activa comprenderán todos los niveles educativos y edad de las mujeres, incluyendo los de Formación Profesional, Escuelas Taller y Casas de Oficios, dirigidos a personas en desempleo, se podrán destinar prioritariamente a colectivos específicos de mujeres o contemplar una determinada proporción de mujeres. 
En cuanto al papel de la negociación colectiva, en el artículo 43 se regula que

De acuerdo con lo establecido legalmente, mediante la negociación colectiva se podrán establecer medidas de acción positiva para favorecer el acceso de las mujeres al empleo y la aplicación efectiva del principio de igualdad de trato y no discriminación en las condiciones de trabajo entre mujeres y hombres.

Por último, respecto a los derechos de conciliación de la vida personal, familiar y laboral, el artículo 44.1 propugna que

se reconocerán a los trabajadores y las trabajadoras en forma que fomenten la asunción equilibrada de las responsabilidades familiares, evitando toda discriminación basada en su ejercicio. 3. Para contribuir a un reparto más equilibrado de las responsabilidades familiares, se reconoce a los padres el derecho a un permiso y una prestación por paternidad, en los términos previstos en la normativa laboral y de Seguridad Social.

Finalmente, en el Estatuto de los Trabajadores, al igual que en la Ley Orgánica de Igualdad, es posible distinguir una vertiente formal y material del principio de igualdad y no discriminación en las relaciones laborales ${ }^{5}$. Así, la vertiente formal se consagra en el artículo 4.1.c) como un derecho básico de los trabajadores: "A no ser discriminados directa o indirectamente para el empleo, o una vez empleados, por razones de sexo...." y en el artículo 17.1 se establece la nulidad de:

los preceptos reglamentarios, las cláusulas de los convenios colectivos, los pactos individuales y las decisiones unilaterales del empresario que den lugar en el empleo, así como en materia de retribuciones, jornada y demás condiciones de trabajo, a situaciones de discriminación directa o indirecta desfavorables por razón de edad o discapacidad o a situaciones de discriminación directa o indirecta por razón de sexo.

Respecto a la segunda vertiente, el artículo 17.4. fija que:

4. Sin perjuicio de lo dispuesto en los apartados anteriores, la negociación colectiva podrá establecer medidas de acción positiva para favorecer el acceso de las mujeres a todas las profesiones. A tal efecto podrá establecer reservas y preferencias en las condiciones de contratación de modo que, en igualdad de condiciones de idoneidad, tengan preferencia para ser contratadas las personas del sexo menos representado en el grupo profesional de que se trate. Asimismo, la negociación colectiva podrá establecer este tipo de medidas en las condiciones de clasificación profesional, promoción y formación, de modo que, en igualdad de condiciones de idoneidad, tengan preferencia las personas del sexo menos representado para favorecer su acceso al grupo profesional o puesto de trabajo de que se trate.

\footnotetext{
5 Sobre las relaciones entre el principio de igualdad de trato en su vertiente formal y material y las diversas implicaciones que tiene en el seno de la empresa véase: Sierra (2011: 216 y ss.).
} 
En este contexto el papel de los planes de igualdad es fundamental para el logro de la igualdad real o material, ya que, al ser de ámbito de empresa o centro de trabajo, son los que mejor pueden detectar las situaciones de discriminación específicas, normalmente de difícil identificación y erradicación, lo que permitirá adoptar las medidas más eficaces para erradicar, o al menos mitigar, dicha discriminación.

\section{La situación actual de la mujer en el mercado laboral con respecto a la brecha de género}

Tal y como ha sido apenas expuesto, la adopción de medidas de igualdad sustancial está plenamente admitidas en el ordenamiento jurídico español y comunitario, siempre que se pueda justificar el por qué de su necesidad ${ }^{6}$. Pues bien, hoy en día en nuestro sistema de relaciones laborales, económicas y sociales existe lo que se conoce como brecha de género en el mercado laboral, que se manifiesta a través de diversos indicadores que permiten identificar los focos de discriminación, lo que legitimará actuaciones concretas en los Planes de igualdad para garantizar la consecución del principio de igualdad sustancial, no solo formal.

Los diversos estudios realizados y los datos obtenidos muestran la siguiente realidad sobre el trabajo femenino respecto de la brecha por género en el empleo. En primer lugar, pese a esta evolución en las últimas décadas, la participa-

6 A nivel internacional, en el Informe de la Organización Internacional del Trabajo (OIT) (2017) se señala que uno de los desafíos más importante a los que se enfrenta en la actualidad el mercado laboral es acabar con la desigualdad de género que se manifiesta principalmente en una menor participación de las mujeres en el mercado laboral y en unas mayores dificultades para encontrar un trabajo, concentrándose la mano de obra femenina en empleos de peor calidad, trabajando más horas en empleos no remunerados y con salarios inferiores ya que trabajan a tiempo parcial o bien porque directamente se les retribuye menos. En especial, el Informe resalta que las mujeres que se quieren incorporar al mercado laboral tienen limitadas sus alternativas profesionales, ya que existen factores como la "la discriminación, la educación, las tareas de cuidado no remuneradas, el equilibro trabajo/vida familiar y la situación matrimonial». A modo de ejemplo resalta que el 20\% de los hombres y $14 \%$ de las mujeres piensan que no es aceptable que una mujer trabaje fuera de su hogar. En este sentido, el Informe propone la necesidad de actuaciones para conseguir la igualdad salarial por trabajos de igual valor; acabar con la segregación profesional y sectorial; actuar sobre el trabajo no remunerado y, en general, acabar con la situación de discriminación laboral de la mujer en el mundo.

Más datos a nivel internacional y comunitario sobre la situación de discriminación de la mujer en el mercado de trabajo que confirman que se trata de un fenómeno global es posible encontrarlos en World Economic Forum. The global gender gap report 2016, http://reports.weforum.org/globalgender-gap-report-2016/; Eurofound, 6th European Working Conditions Survey, Luxembourg, 2016. https://www.eurofound.europa.eu/es/publications/report/2016/working-conditions/sixth-europeanworking-conditions-survey-overview-report y Occupational change and wage inequality: European Jobs Monitor 2017. https://www.eurofound.europa.eu/publications/report/2017/occupational-changeand-wage-inequality-european-jobs-monitor-2017, Luxembourg, 2017. 
ción laboral femenina sigue siendo significativamente más baja que la masculina, tanto en España como en los países de la Unión Europea, sobre todo en hogares con parejas e hijos. Así, la tasa de actividad de las mujeres de 16 a 64 años de edad era, en el conjunto UE-28 en 2015, un 14,9\% inferior a la de los varones, siendo en España la diferencia de un 13,5\%. En el tramo de 25 a 64 años la distancia europea era un $15,5 \%$ y la española un $14,2 \%{ }^{7}$.

En segundo lugar, la tasa de empleo femenina es menor. Así, en el cuarto trimestre de 2015, la tasa de actividad masculina era un 21,5\% mayor que la femenina, y la tasa de empleo era un $26,3 \%$ mayor. Ello, a su vez, conlleva que su tasa de paro sea más elevada. En concreto, la tasa de paro masculina es dos puntos inferior a la femenina. En este sentido, una de las características distintivas de la evolución reciente del mercado de trabajo es que la tasa de empleo femenina acumula un aumento de 2,9 puntos en el bienio 2013-2015, frente a 4,2 puntos en la masculina. A su vez, entre 2013 y 2015 el paro femenino en España se redujo 4,0 puntos y el masculino 5,6 puntos, aunque las tasas de parados de larga duración eran similares (CES, 2016: 50 y ss.).

En cuanto a la brecha salarial, no solo se manifiesta en salarios menores sino que también afecta a las prestaciones de la seguridad social a través de las bases de cotización puesto que a menor remuneración menor cotización ${ }^{8}$. El punto de partida debería de situarse en la segregación por sexos en el nivel educativo y en la formación de las trabajadoras de cara a su incorporación al mercado laboral ${ }^{9}$. A su vez, existe una mayor presencia de las mujeres en ocupaciones peor remuneradas por varias razones. En primer lugar, el coste de oportunidad de salir o permanecer fuera del trabajo es menor para las mujeres por la brecha salarial, lo que supone que, en caso de necesidad para una familia, sean ellas las que opten

\footnotetext{
${ }^{7}$ Consejo Económico y Social (CES) (2016: 39 y ss). El Informe pone de relevancia cómo dicha participación laboral va unida al nivel de estudios, tipo de estudios cursados y de edad, pero fundamentalmente al estado civil y circunstancias personales. Así, en el grupo de 20 a 49 años las mayores tasas de participación laboral corresponde a los hogares unipersonales, a los monoparentales y a los formados por una pareja sin hijos. Los primeros mostraban en 2015 tasas de actividad cercanas al $95 \%$ y los segundos en torno al 90\%. En este mismo grupo de edad las menores tasas de actividad correspondían a las mujeres en hogares compuestos por una pareja e hijos, siendo más bajas cuando el hijo más pequeño tenía menos de 3 años de edad. Asimismo, el Estudio resalta que, aunque las trabajadoras universitarias tenían tasas de actividad más altas, la distancia entre las mayores tasas (mujeres solas o sin hijos) y las menores (mujeres en pareja con hijos pequeños) era casi igual que en el total de mujeres por nivel de estudios.

${ }^{8}$ En general, sobre la brecha salarial y la discriminación retributiva en nuestro país véase: Figueroa y Segovia (2016); Pons (2015: 11 y ss.) y Cabeza (2012: 81 y ss.)

9 CES (2016: 45 y ss). Mientras que en los hombres la tasa de actividad es alta, incluso en los niveles de estudios básicos, en las mujeres crece con fuerza en los secundarios profesionales y, sobre todo, en los universitarios, donde se iguala a la de los varones $(81,4$ y $81,2 \%$, respectivamente). Otro dato a tener en cuenta es que la presencia femenina es más baja en las denominadas STEM (ciencia, tecnología, ingeniería y matemáticas), que tienen una mayor demanda en la sociedad del conocimiento y que, de por sí, son cursadas con menor frecuencia en España.
} 
por permanecer en el hogar ${ }^{10}$. En segundo lugar, existe una amplia polarización del trabajo femenino, con menor peso de ocupaciones intermedias respecto del masculino y mayor peso en las asociadas con bajas cualificaciones (CES, 2016. 58) y, en tercer lugar, el mayor uso de las mujeres de las medidas de conciliación, debida a la desigual distribución de las cargas familiares de cuidados y de tareas domésticas, se traduce en interrupciones más frecuentes en su vida laboral, lo que afecta a su remuneración (CES, 2016: 121 y ss.) ${ }^{11}$.

Pues bien, a pesar de todas las reformas llevadas a cabo, tanto a nivel constitucional como legal, hoy por hoy la brecha salarial global entre hombres y mujeres en el mercado laboral español alcanza $23,25 \%{ }^{12}$. Las principales causas de la actual situación son principalmente tres: una menor retribución por hora trabajada; menores tasas de empleo femenino, debido fundamentalmente a la interrupción de las carreras profesionales para la atención y cuidado de los hijos, $\mathrm{y}$ el hecho de que las mujeres trabajan menos horas que los varones en trabajos retribuidos. En cuanto a los factores que contribuyen a esta situación son varios (OIT, 2017: 2). Así, los puestos de altos cargos y directivos en las empresas son ocupados principalmente por varones, lo que facilita su promoción y salarios más altos; existe una mayor presencia de la mujer en actividades no retribuidas y una mayor tasa de trabajo a tiempo parcial; prevalece una segregación en la edu-

10 CES (2016: 57 y ss). Estos datos son consecuencia de una desigual atribución de valor a los trabajos más feminizados y del reparto y asunción de tareas de cuidados por género, en el marco de un sistema que impulsa la asunción de estos roles por las mujeres. A su vez, el empleo asalariado es mayor en las mujeres que en los hombres, ya que hay menos empresarias en todas las categorías. En 2015 , los asalariados eran el 79,1\% en el total del empleo masculino, mientras llegaban al $87,4 \%$ en el femenino, mientras que los empleadores eran un 6,6 y un 3,3\%, respectivamente, y el empleo autónomo era un $13,8 \%$ entre los hombres y un $8,6 \%$ entre las mujeres.

11 El CES resalta que compartir el permiso de maternidad es algo residual hoy en día. Así, según la encuesta de la UAM, el 80\% de las madres trabajadoras pide el permiso y, en su inmensa mayoría, ellas agotan su duración. La mayor parte de las que opta por no disfrutar del permiso son trabajadoras por cuenta propia. En cambio, únicamente el 5\% de los padres con este derecho han utilizado parte del permiso de maternidad, en su mayoría asalariados, un porcentaje que se limita a algo más del $2 \%$ respecto del total. En cuanto al permiso de paternidad los datos anteriores al ańo 2017 indican que lo disfrutó el $75 \%$ de los beneficiarios, siendo su utilización más frecuente cuando ambos progenitores trabajan y más habitual entre los asalariados y entre los trabajadores del sector público. Por lo que se refiere a las excedencias, los datos ponen de manifiesto que, a pesar de que tanto hombres como mujeres tienen derecho a usarlo, son mayoritariamente las trabajadoras quienes se acogen a ellos, generalmente inmediatamente después del permiso de maternidad. Concretamente, en 2015 solo el 6,7\% de las excedencias por cuidado de hijos y el 15,4\% de las excedencias por cuidados familiares fueron disfrutadas por hombres. Un dato relevante es que, tras la finalización del período de disfrute de las mismas, el $100 \%$ de los varones vuelven a trabajar a tiempo completo mientras que en el caso de las mujeres se reduce al $55 \%$, y, además, reducen su jornada o trabajan a tiempo parcial (35\%), abandonan el mercado (7\%),o pierden el empleo (3\%). Respecto a la reducción de la jornada solo el 3,3\% de los varones con al menos un hijo menor de 8 ańos redujo su jornada, frente al $22,5 \%$ en el caso de las mujeres. Por otra parte, el $93 \%$ de las mujeres alargaron el permiso por más de un mes, mientras que este porcentaje se limita al 60\% en el caso de los hombres.

12 http://www.expansion.com/economia/2017/08/05/5985c190ca474128698b4570.html. 
cación que, a su vez, produce una segregación en las ocupaciones laborales que se refleja en salarios menores en aquellas tareas consideradas tradicionalmente femeninas y, por último, la discriminación salarial directa también contribuye a la diferencia de remuneración (OIT, 2017: 4) ${ }^{13}$.

Por lo que se refiere a la brecha en sus trayectorias laborales y carreras profesionales, las mujeres presentan mayores tasas de temporalidad y parcialidad unida principalmente a un reparto no equitativo de las tareas domésticas y de cuidados, cuya manifestación es el mayor uso que realizan de los instrumentos de conciliación laboral en cuanto a suspensiones, excedencias y permisos, lo que supone una menor vinculación con el trabajo y unas peores condiciones laborales (CES, 2016: 63 y ss.).

En primer lugar, el contrato a tiempo parcial es un contrato femenino, tal y como lo demuestran las cifras. A modo de ejemplo, el $72 \%$ del empleo a tiempo parcial se desempeña por mujeres, datos que en el sector servicios puede ascender al 75\% — 901.126 mujeres frente a 1.006 .836 varones-. En cambio, las cifras del trabajo a tiempo completo se invierten - 5.046.113 hombres por 3.489.752 mujeres-. Las trabajadoras afiliadas a tiempo parcial son casi dos tercios de los contratos a tiempo parcial frente al $41 \%$ de los contratos a tiempo completo y suponen un $61,65 \%$ de los trabajos indefinidos y un $58,11 \%$ de los trabajos temporales. En 2015 unos 53 contratos fueron firmados por mujeres a tiempo completo frente a 73 suscritos por los trabajadores. Globalmente, los contratos de las quince ocupaciones con mayor contratación femenina en la modalidad de interinidad representan cerca del $73 \%$ en 2015 con una jornada parcial del 45,61\% (SEPE, 2017: 33 y ss.). Otros datos a tener en cuenta es la mayor concentración del empleo a tiempo parcial en actividades de baja productividad y la involuntariedad que en España se sitúa alrededor del 60,1\% (CES, 2016: 65 y ss.).

En segundo lugar, la tasa de temporalidad en 2015 fue del 25,8\% para las mujeres y del $25,6 \%$ para los hombres, aunque entre los jóvenes eran mayores para el género femenino (86,2 y 81,8\% en el grupo de 16 a 19 años, y 70,3 y $68,2 \%$ en el de 20 a 24 años), así como, aunque en menor medida, en los grupos a partir de 40 años, si bien es cierto que estos datos han de ser interpretados en un contexto de inestabilidad en el empleo, con una mayor rotación entre empleo y paro, por lo que la menor tasa de temporalidad actual y la igualación entre sexos de ella, no implica, por sí misma, una mejora relativa de las condiciones de trabajo femenino (CES, 2016: 65 y ss.).

13 Respecto al impacto de la segregación profesional y la segmentación laboral que tiene su origen en la negociación colectiva, fundamentalmente en los sistemas de clasificación profesional, véase López (2015: 579 y ss.) y Garrigues (Dir.) (2017). 


\section{El papel de la negociación colectiva en general y de los planes de igualdad en particular en la consecución del principio de igualdad sustancial}

\subsection{Delimitación de las funciones en materia de igualdad}

El papel fundamental de los planes de igualdad pasa, en primer lugar, por identificar dónde están los focos de discriminación real en una empresa para poder, en segundo lugar, garantizar la adopción de medidas eficaces para la consecución del principio de igualdad sustancial, no solo formal, y erradicar los efectos de la misma ${ }^{14}$. De hecho, el artículo 46 LOI así los define y delimita:

1. Los planes de igualdad de las empresas son un conjunto ordenado de medidas, adoptadas después de realizar un diagnóstico de situación, tendentes a alcanzar en la empresa la igualdad de trato y de oportunidades entre mujeres y hombres y a eliminar la discriminación por razón de sexo. Los planes de igualdad fijarán los concretos objetivos de igualdad a alcanzar, las estrategias y prácticas a adoptar para su consecución, así como el establecimiento de sistemas eficaces de seguimiento y evaluación de los objetivos fijados.

Es importante resaltar esta función, puesto que el papel de la negociación colectiva, como instrumento de mejora de las condiciones laborales en materia de igualdad de derechos en el mercado laboral, hasta el momento ha consistido fundamentalmente en declaraciones o reconocimiento formal de derechos, en muchas ocasiones reproduciendo, a veces incluso con errores o de manera incompleta, la normativa estatal y estatutaria sobre esta cuestión más que en establecer derechos efectivos y reales, acompañados de medidas eficaces, que hayan eliminado o reducido de manera significativa la brecha de género del mercado laboral. En este sentido, las áreas prioritarias de actuación se han centrado en temas relacionados con la maternidad y atención y cuidado de hijos o familiares, desde una perspectiva de que el destinatario es la trabajadora y perpetuando de alguna manera los roles tradiciones del trabajo femenino, más que de introducir cambios en la concepción misma de ese rol que ayuden a superar las condiciones sociales que dan lugar a la discriminación que no se puede erradicar y que formarían parte del principio de igualdad sustancial, en cuanto remoción de las desigualdades del artículo 9.2 CE.

A este respecto, las competencias de la negociación colectiva — los planes de igualdad son una manifestación de la misma- quedan claras desde que, por un

14 En general, sobre el papel de los Planes de Igualdad en el desempeño de sus funciones véase Luján y Selma (2016: 455 y ss.) y Mercader y Pérez del Prado, (2010: 179 y ss.). Para una propuesta de un modelo de aplicación práctica de los planes de igualdad véase: Romero (2017). 
lado, el artículo 64.3 ET, dentro de los derechos de información y consulta y competencias de los representantes de los trabajadoras, señala que:

3. También tendrá derecho a recibir información, al menos anualmente, relativa a la aplicación en la empresa del derecho de igualdad de trato y de oportunidades entre mujeres y hombres, entre la que se incluirán datos sobre la proporción de mujeres y hombres en los diferentes niveles profesionales, así como, en su caso, sobre las medidas que se hubieran adoptado para fomentar la igualdad entre mujeres y hombres en la empresa y, de haberse establecido un plan de igualdad, sobre la aplicación del mismo.

Y, por otro lado, el artículo 85.1 ET, en el apartado de contenido de los convenios colectivos, establece el deber de negociar medidas de igualdad:

Sin perjuicio de la libertad de las partes para determinar el contenido de los convenios colectivos, en la negociación de los mismos existirá, en todo caso, el deber de negociar medidas dirigidas a promover la igualdad de trato y de oportunidades entre mujeres y hombres en el ámbito laboral o, en su caso, planes de igualdad con el alcance y contenido previsto en el capítulo III del título IV de la Ley Orgánica 3/2007, de 22 de marzo, para la igualdad efectiva de mujeres y hombres.

Ya dentro del texto de la LOI, el artículo 45 establece la obligación de las empresas de:

respetar la igualdad de trato y de oportunidades en el ámbito laboral y, con esta finalidad, deberán adoptar medidas dirigidas a evitar cualquier tipo de discriminación laboral entre mujeres y hombres, medidas que deberán negociar, y en su caso acordar, con los representantes legales de los trabajadores en la forma que se determine en la legislación laboral.

Por lo que se refiere al concreto papel de los planes de igualdad es muy amplio en cuanto a su contenido. Así, el artículo 46.3 LOI señala las concretas áreas de actuación, citando a modo de ejemplo ya que se trata de un listado abierto:

acceso al empleo, clasificación profesional, promoción y formación, retribuciones, ordenación del tiempo de trabajo para favorecer, en términos de igualdad entre mujeres y hombres, la conciliación laboral, personal y familiar, y prevención del acoso sexual y del acoso por razón de sexo.

El problema es que no todas las estrategias que se adopten para erradicar la brecha de género pueden responder a un mismo modus operandi, puesto que, si bien la desigualdad estructural de las mujeres en el mercado laboral es consecuencia de un patrón social de discriminación muy homogéneo en cuanto a sus causas, la igualdad sustancial no se alcanza con un modelo único de actuación, 
sino que cada área o eje del Plan de igualdad, probablemente, demande medidas de distinto alcance e intensidad, aunque complementarias entre sí, dando cumplimiento al principio de transversalidad, dependiendo de a qué ámbitos de la relación laboral se refiera, por lo que es fundamental un correcto diagnóstico en cada empresa o centro de trabajo para establecer unas medidas u otras. Así, no es lo mismo idear acciones concretas para acabar con la desigualdad retributiva origen de la brecha salarial, que establecer medidas de corresponsabilidad para el disfrute de los derechos de atención a la familia para acabar, por ejemplo, con la alta tasa de parcialidad del trabajo femenino. Pues bien, teniendo en cuenta que la legislación habilita y fomenta que en el contenido de los Planes de igualdad se contemplen actuaciones encaminadas a la consecución del principio de igualdad sustancial se hace necesario un estudio de las mismas.

\subsection{Análisis de planes de igualdad y convenios colectivos desde la perspectiva del principio de igualdad sustancial}

En primer lugar, el artículo 46 del I Convenio colectivo sectorial de limpieza de edificios y locales (BOE 23 mayo 2013) regula las cuestiones relacionadas con el principio de igualdad de trato y no discriminación. Así, se contemplan medidas de acción positiva en cuanto a tratamientos preferenciales en el empleo, eso sí, a favor del sexo menos representado, en distintos ámbitos y momentos de la relación laboral: ingreso, contratación, formación y promoción, y conversión de contratos temporales en indefinidos. También contempla aspectos relacionados con los criterios de selección desde una perspectiva no discriminatoria; el establecimiento de cuotas en los puestos de responsabilidad o la erradicación desigualdad retributiva oculta originada durante el disfrute del permiso por maternidad, indicando que todos estos aspectos se incorporen al Plan de igualdad ${ }^{15}$.

15 «Las partes firmantes del presente Convenio Colectivo Sectorial adoptan los siguientes Principios Generales en orden a la no discriminación e igualdad de trato de todos los trabajadores/trabajadoras. Estos Principios Generales son: 1. A la preferencia para el ingreso del género menos representado sin que ello se haga en detrimento de los méritos e idoneidad de otros trabajadores. 2. A la fijación de criterios que favorezcan la contratación, formación y promoción del género menos representado en el grupo profesional de que se trate. 3. A La fijación de criterios de conversión de contratos temporales a fijos que favorezcan al género menos representado, en igualdad de condiciones y méritos, se encuentren afectadas en mayor medida que los hombres por el nivel de temporalidad. 4. Al estudio y, en su caso, el establecimiento de sistemas de selección, clasificación, promoción y formación, sobre la base de criterios técnicos, objetivos y neutros por razón de género. 5. Al establecimiento de cuotas en términos de porcentajes de mujeres y hombres para lograr una distribución más equilibrada en los puestos de responsabilidad. 6. A recoger que las ausencias debidas a la maternidad no perjudiquen a las trabajadoras a la hora de percibir determinados pluses (permanencia, asistencia, etc.) y algunos complementos salariales que pueden ser fuente de discriminación (disponibilidad, antigüedad, etc.). 7. A la inclusión de un módulo de igualdad en el plan de formación anual de la empresa. 8. A determinar unos objetivos generales de igualdad a alcanzar e la empresa, las materias, las fases, los plazos y la estructura básica del plan. 9. A incorporar en los planes de igualdad los contenidos minimos que introduce el proyecto de ley referidos al acceso al empleo y la no segregación ocupa- 
En segundo lugar, existen convenios colectivos que regulan esta cuestión desde una perspectiva transversal, afectando a todas las políticas de la empresa, señalando actuaciones específicas y distintas para cada materia, como son la selección, formación, promoción profesional, retribución y prevención del acoso. Así, el II Convenio colectivo profesional de los controladores de tránsito aéreo en la Entidad Pública Empresarial Aeropuertos Españoles y Navegación Aérea (BOE 9 marzo 2011), en el apartado selección y promoción profesional, exige que los criterios a aplicar sean neutros o bien que en las vacantes se facilite la incorporación de mujeres si están infrarrepresentadas, especificando las medidas para ello. También se señala la necesidad de tener en cuenta a los trabajadores con los contratos suspendidos por el ejercicio de los derechos de conciliación en los procesos de promoción. En cuanto a la desigualdad retributiva, limita los complementos ad personam para erradicarla ${ }^{16}$.

cional, la promoción, la formación, la igualdad retributiva, la conciliación de la vida personal, familiar y laboral y la formación especifica en materia de igualdad entre trabajadores. 10. Las empresas deberán promover condiciones de trabajo que eviten el acoso sexual y el acoso por razón de sexo y arbitrar procedimientos especificos para su prevención y para dar cauce a las denuncias o reclamaciones que puedan formular quienes hayan sido objeto del mismo».

16 "Artículo 215. Medidas tendentes al favorecimiento de la igualdad de trato y de oportunidades entre mujeres y hombres. El principio de igualdad efectiva entre mujeres y hombres y su correlato de no discriminación por razón de género informará con carácter transversal todas las actuaciones de AENA en el marco de este II Convenio Colectivo, y estará presente en cualesquiera de sus politicas de Recursos Humanos, especialmente en aquellas que hagan referencia a los procesos de selección, formación, promoción profesional, retribución y prevención del acoso laboral, sexual y del acoso por razón de sexo. Al objeto de hacer efectivo el principio de igualdad y no discriminación a que hace referencia el punto anterior, AENA y USCA pondrán en marcha las actuaciones necesarias para la consecución de los siguientes objetivos:1. En materia de selección y promoción profesional: 1.1 Garantizar la inexistencia, en las bases de las convocatorias de selección y promoción, asi como de cambio de destino, de cualquier requisito relacionado directa o indirectamente con el sexo. 1.2 Facilitar la incorporación de las mujeres en las vacantes que se produzcan en aquellos puestos de trabajo en los que se encuentren subrepresentadas, con especial atención a los puestos de gestión y en las comisiones paritarias, en los que se utilizará, siempre que sea posible, un sistema de cupos iguales. A estos efectos se adoptarán, entre otras, las siguientes medidas: ${ }^{*}$ Utilización de canales de comunicación y difusión que faciliten la captación de personal femenino: universidades con mayores porcentajes de mujeres, asociaciones, colegios profesionales o instituciones dedicadas a la promoción e impulso de la mujer. * Informar, formar y motivar a las mujeres para que participen en procesos de selección o promoción a puestos de trabajo tradicionalmente masculinos. 1.3 Posibilitar la participación de las trabajadoras y trabajadores, en situación de suspensión de contrato por maternidad o paternidad, excedencia por motivos familiares con derecho a reserva de puesto. 2. En materia de formación: Facilitar el acceso a la formación de las trabajadoras y trabajadores en situación de disminución de jornada por cuidado de hijos, familiares enfermos y/o discapacitados, excedencia por motivos familiares y suspensión de contrato por maternidad o violencia de género. 3. En materia de retribuciones: Garantizar, en aquellos conceptos retributivos que queden a discreción exclusiva de AENA, la aplicación efectiva del principio de igual remuneración por un trabajo de igual valor y, en concreto, la no existencia de diferencias salariales por razón de género. A tal efecto se entiende que, a igualdad de condiciones laborales en lo referente a dependencia, antigüedad, puesto de carrera profesional y condiciones personales, las diferencias retributivas sólo estarán justificadas por aquellos complementos que con carácter "ad personam» tengan su justificación en situaciones derivadas de anteriores normativas reguladoras. 4. En materia de prevención del acoso sexual y del acoso por razón de sexo: En el plazo máximo de tres meses a partir de la entrada en vigor del presente Con- 
En tercer lugar, hay convenios colectivos que prestan especial atención a establecer medidas que garanticen la igualdad formativa y en el desarrollo de competencias y habilidades que favorezcan la promoción y desarrollo profesional. Incluso se fijan medidas concretas en cuanto a número de mujeres que han de ser promocionadas en un período concreto de tiempo ${ }^{17}$. Así:

B. Formación. La empresa y las representaciones sindicales comparten el papel fundamental que tiene la formación en el desarrollo de una cultura igualitaria. A fin de facilitar la evolución de la mujer en aquellos niveles profesionales en los que se encuentre menos representada se elaborarán programas de formación orientados a desarrollar las competencias en las que se observe un mayor desequilibrio. En todo programa formativo impartido en la empresa se propiciará la participación equilibrada de género, al menos en proporción a su presencia en las áreas afectas al programa formativo. C. Promoción y desarrollo profesional. Para contribuir al equilibrio progresivo de la menor representación de la mujer en los niveles profesionales del I al V, el Banco adquiere el compromiso de potenciar el desarrollo de competencias y habilidades que impulsen y aceleren su incorporación a puestos de mayor responsabilidad, utilizando a tal fin las mejores herramientas y prácticas de recursos humanos. De acuerdo con lo establecido en el artículo 54.4 del CCB y atendiendo a la información de seguimiento del Plan de Igualdad a 31-12-2015 en lo que refiere a plantilla por categorías y niveles, se establece que para el periodo comprendido desde el 1-62016 hasta 31-12-2020, se promocionará como mínimo a 300 mujeres a nivel VII y como mínimo a 150 mujeres a nivel $\mathrm{V}$, en aras a lograr una composición equilibrada en dichos niveles profesionales.

En cambio, la política empresarial se muestra más opaca en lo referido a las medidas de igualdad retributiva, en la que se señala:

D. Retribución salarial. No obstante, y con el fin de asegurar la objetividad en la retribución y evitar que se produzcan desviaciones por razón de género, el Banco ha establecido los controles internos necesarios para que a similar trayectoria profesional, desarrollo personal y mérito en el desempeño de un mismo puesto funcional, las retribuciones sean equivalentes.

En cuarto lugar, hay planes que exigen un diagnóstico de la situación de la empresa como condición previa para adoptar este tipo de medidas. Así, el Plan

\footnotetext{
venio, AENA y USCA, a través de la Comisión Paritaria de Igualdad, elaborarán un código de buenas prácticas en esta materia, para su aprobación en el seno de dicha Comisión y su difusión al conjunto de la plantilla de CCAs. Sin perjuicio de lo establecido en la normativa de régimen disciplinario, se garantizará, tanto por AENA como por USCA, el tratamiento reservado de las denuncias de hechos que pudieran ser constitutivos de acoso sexual o de acoso por razón de sexo. 5. En materia de información, comunicación y sensibilización: En todas las comunicaciones e informaciones de AENA, así como en sus actos públicos de divulgación, publicidad y reclutamiento, se tendrá especial cuidado en la utilización de un lenguaje neutro $y$ no sexista".

17 Plan de igualdad del Banco de Sabadell, BOE, de 11 de agosto de 2017.
} 
de igualdad de Comedores colectivos de Celis, SL (BOE, de 14 de junio de 2017) establece que:

Para la realización del diagnóstico de este Plan de Igualdad, y dada la actividad principal de la empresa ligada al servicio de comida en los comedores escolares, se han observado los datos existentes en la empresa durante el pasado curso escolar 2015-2016 y especialmente datos del mes de noviembre de 2015, que ha sido tomado como mes de referencia dado que durante el mismo la actividad de la empresa es más lineal y se encuentra a pleno rendimiento. El presente Plan de Igualdad parte del hecho de que en la actividad desarrollada por Comedores Colectivos de CELIS, S.L., existe una importante feminización de las profesiones, siendo el sexo masculino el que está presente en todas las categorías profesionales.

Es de resaltar cómo este Plan de igualdad tiene en cuenta la situación de subrogación con un elemento que dificulta la consecución de la igualdad real:

La garantía del mantenimiento de los puestos de trabajo existente en el sector de restauración colectiva supone que la mayoría de la plantilla se incorpora a Comedores Colectivos de CELIS, S.L., por el ejercicio del derecho y del deber de la subrogación, por lo que la empresa no puede cambiar tendencias en los porcentajes de contratación por categorías profesionales. Respecto al personal administrativo, técnico y gestor de la empresa, así como todo aquel personal que se incorpore inicialmente a la plantilla de la empresa, se promueve la promoción interna en el acceso al empleo, y solo cuando no es posible, se acude a la contratación externa.

A su vez, dentro de los objetivos generales se incide en la necesidad de:

3. Asegurar que la gestión de Recursos Humanos es conforme a los requisitos legales aplicables en materia de igualdad de oportunidades» y de «4. Reforzar el compromiso responsabilidad social corporativa asumido por Comedores Colectivos de CELIS, S.L.

En quinto lugar, el Plan de igualdad de Serveis Integrals Lafuente (BOE de 27 de abril de 2017) es un buen ejemplo de cómo se deben elaborar las medidas concretas del Plan de igualdad, a partir de los datos de una plantilla que contiene con las contrataciones realizadas en el último año.

1. Área de acceso al empleo. Objetivo 1: «Sensibilización de las personas responsables de los procesos de selección por medio de acciones formativas». Una vez realizado el diagnóstico, las cifras nos muestran una realidad de empresa especial donde no es necesario fomentar la igualdad de oportunidades de las mujeres, dado que ya existe. De hecho, las cifras demuestran el tanto por cien elevadísimo de mujeres respecto a los hombres, en casi todos los puestos existentes y a todos los niveles. Por tanto, no es necesario aumentar la participación en puestos de representación, pero Serveis Integrals Lafuente SL, se ha decidido marcar como objetivo en esta área, la sensibilización de las personas responsables de los procesos de selección, en un in- 
tento de analizar porque los hombres no tienen presencia en el sector empresarial en el que estamos incluidos, y para que en los procesos de selección estén siempre garantizada la ausencia de barreras que discriminen a los/as candidatos/as por razón de sexo. Objetivo 2: «Fomentar el acceso y promoción de la mujer en los sectores y categorías profesionales menos representados» Según los datos de nuestro diagnóstico, la mujer está subrepresentada porcentualmente en las categorías profesionales "conductor/a-limpiador/a» y "peón especializado» y "especialista», por lo que la empresa buscará formas de acceso de las mujeres en plantilla a cursos relacionados con estas temáticas, para su posible promoción profesional. Área de clasificación profesional, promoción y formación. Respecto a la clasificación profesional, Serveis Integrals Lafuente cuenta con una detallada descripción de los puestos de trabajo y sus competencias en los diferentes convenios colectivos de aplicación. Respecto a la promoción profesional, nuestro objetivo será: «Velar por el mantenimiento de la cultura empresarial que fomenta la promoción interna y la comunicación abierta sobre la disponibilidad de puestos.» Respecto a la formación el objetivo en este ámbito será: «Velar por una planificación de la formación que facilite la asistencia de toda la plantilla y suponga una mejora profesional para las mujeres en las categorías menos representadas». Ârea de retribuciones En esta área Serveis Integrals Lafuente, SL, mantiene un perfecto equilibrio de igualdad de oportunidades entre mujeres y hombres. Así pues, podemos concluir que no es necesario marcarse un objetivo de fomento de la igualdad salarial de las mujeres, dado que existe en la actualidad y que no hay barreras para el empleo femenino. No obstante, y dada la necesaria sensibilización del total de la plantilla en todo este asunto, sí vemos oportuno marcarnos un objetivo claro encaminado a sensibilizar y a mantener en el tiempo el logro que en la actualidad hemos alcanzando, evitando riesgos en el futuro. Así, en esta área el objetivo será: «Velar por la igualdad de oportunidades entre mujeres y hombres y fomentar las acciones de responsabilidad social en la empresa.

Este mismo Plan señala otras áreas de actuación desde la perspectiva de la igualdad sustancial, de las que merece destacar las medidas de corresponsabilidad:

Sensibilizar a la plantilla de la empresa de la necesidad de fomentar la igualdad entre hombres y mujeres, la corresponsabilidad y la conciliación de la vida personal, familiar y laboral. El significado de la corresponsabilidad social va más allá de aumentar la implicación de las personas en el reparto de las responsabilidades domésticas y familiares, especialmente los hombres, para extenderse a otros agentes sociales e instancias públicas y privadas.

En sexto lugar, ya hay planes de igualdad que parten del análisis de los resultados obtenidos por planes anteriores para poner en marcha propuestas de mejora a partir de los mismos. Así, el Plan de igualdad de Activa Mutua 2008 (BOE de 18 de enero de 2017) considera el Plan como una estrategia más de la empresa que se integra en cada una de sus actividades y procedimientos, dentro del marco de la responsabilidad social corporativa. A modo de ejemplo cabe citar la propuesta de gestión organizativa igualitaria: 
2. Gestión organizativa igualitaria. 8. Avanzar en el fomento de la incorporación de mujeres en los puestos de responsabilidad de la empresa, tanto a través de futuras incorporaciones como a través de promociones internas en la organización. 9. Trabajar en la obtención de datos segregados por territorio y área, departamento o puesto de trabajo, con la finalidad de poder identificar sectores especialmente masculinizados o feminizados en la organización, con el objetivo de poder impulsar medidas concretas y de carácter más específico para continuar avanzando en la paridad en cuestión de género. 10. Continuar avanzando en la obtención de información segregada del nivel de formación de todo el personal administrativo. 11. Evaluar la posibilidad de fomentar, en el caso que se lleven a cabo nuevas incorporaciones de personal con algún tipo de discapacidad, la incorporación prioritaria de mujeres.

El propio Plan indica más adelante las acciones concretas a poner en marcha, como puedan ser el fomento de la representación paritaria de manera transversal en toda la organización mediante la incorporación de las mujeres en puestos de trabajo de responsabilidad, tanto si se trata de nuevas incorporaciones como de procesos de promoción interna; la identificación de sectores especialmente masculinizados y feminizados y la obtención de información segregada del nivel de formación del personal administrativo.

En último lugar, el Plan de igualdad de Centro de Seguros y Servicios, Correduría de Seguros El Corte Inglés, SA, BOE, de 19 de enero de 2017, resalta especialmente la apuesta por las medidas de acción positiva con el fin de:

hacer efectivo el derecho constitucional de la igualdad, las personas físicas y jurídicas privadas podrán adoptar medidas específicas a favor de las mujeres para corregir situaciones patentes de desigualdad de hecho respecto de los hombres. Tales medidas, que serán aplicables en tanto subsistan dichas situaciones, habrán de ser razonables y proporcionadas en relación con el objetivo perseguido en cada caso.

También se fija actuaciones específicas en materia de selección para la eliminación de los desequilibrios por sexo que dan lugar a la segregación horizontal y vertical, mediante la diversificación profesional e impulsando medidas de acción positiva en la política de selección que:

posibiliten un mayor equilibrio y una mayor equiparación de los géneros. Para ello, en igualdad de condiciones de idoneidad y competencia se seleccionará para el puesto una mujer cuando se trate de áreas y puestos masculinizadas de la Empresa (...). En igualdad de condiciones de idoneidad y competencias se seleccionarán mujeres para los puestos de áreas masculinizadas.

En relación con esta cuestión, el Plan ahonda en la necesidad de:

Formar en materia de igualdad a la totalidad de las personas que participan en los procesos de selección para la contratación y promoción con el fin de garantizar la objetividad del proceso y evitar actitudes discriminatorias; de manera que los candi- 
datos y candidatas sean valorados/as únicamente por sus cualificaciones, competencias, conocimientos y experiencias sin considerar su sexo.

\section{Propuestas de actuación para los planes de igualdad desde la perspectiva del principio de igualdad sustancial}

El papel de los planes de igualdad para la consecución de la igualdad real en la empresa es fundamental aunque no se puede obviar que está condicionado por la necesidad de que se adopten medidas legislativas complementarias, tal y como ya ha sido señalado. En este sentido, actuar sobre el problema que se plantea con la alta subcontratación y externalización en sectores fuertemente feminizados, acabar con la alta tasa de parcialidad de la mano de obra femenina o reconocer unos derechos de conciliación igualitarios desde la perspectiva de género ${ }^{18}$, que pasen por diferenciar la titularidad de la maternidad biológica del resto de situaciones y no reproduzcan roles sociales desigualitarios, no es una tarea que se pueda encomendar exclusivamente a los Planes de igualdad y a la negociación colectiva pero sí que podrían paliar estas situaciones ideando acciones como, por ejemplo, establecer ayudas a guarderías, la estabilización de la plantilla femenina (Martínez, 2015: 411), el control de las empresas consultoras que participan en los procesos de externalización o el reconocimiento de los derechos salariales a todos los supuestos de suspensión por cuidado de un hijo y no solo a la maternidad biológica o lactancia (Ministerio de Sanidad, Servicios Sociales e Igualdad, 2017).

A pesar de ello, en el contenido de los Planes de igualdad sí que es posible encontrar regulaciones que pueden ayudar a la erradicación de la situación de desigualdad laboral entre sexos, con un contenido novedoso y que pueden servir de pautas de actuación para el futuro. En este sentido, el Plan de igualdad del Banco Sabadell, en el apartado del Código de conducta, integra la erradicación de la discriminación como un instrumento de la promoción de la salud desde una vertiente dinámica e innovadora:

18 En CES (2016: 127 y ss.) se señala expresamente la necesidad de que el sistema de permisos laborales español evolucione hacia: «el reconocimiento de licencias familiares individuales e intransferibles para el padre y la madre, retribuidas, flexibles en cuanto a su disfrute y cuya duración no sea superior a un año para evitar los efectos negativos sobre la situación en el mercado de trabajo, la trayectoria laboral y la cualificación de los progenitores, lo que aconseja también acompañarlas con políticas activas que preserven la empleabilidad de los trabajadoresn. También indica que esta política de permisos debe complementarse con el fortalecimiento de la red de servicios públicos de atención a la infancia y la dependencia que garantice un equilibrio entre el desempeńo del trabajo y la cobertura adecuada de las necesidades de cuidados familiares, al amparo de lo dispuesto en el artículo 44.1 LOI que establece que: «los derechos de conciliación de la vida personal, familiar y laboral se reconocerán a los trabajadores y las trabajadoras en forma que fomenten la asunción equilibrada de las responsabilidades familiares, evitando toda discriminación basada en su ejercicio». 
promover ambientes de trabajo en los que se trate con respeto y dignidad a las personas, procurando el mayor desarrollo profesional de sus empleados y empleadas y garantizando la igualdad de oportunidades en su contratación, formación y promoción, ofreciendo un entorno de trabajo libre de cualquier forma de discriminación o de hostigamiento. Los integrantes de su plantilla tienen derecho a trabajar en un ambiente laboral en el que se promueva la igualdad de oportunidades y en el que no se toleren las prácticas discriminatorias, entre las que se incluye el acoso.

En segundo lugar, hay que destacar el papel fundamental de las Comisiones de seguimiento de los Planes de igualdad por las competencias que se les pueden atribuir para el logro efectivo de los objetivos del Plan ${ }^{19}$. Así, el Plan de igualdad del Banco Sabadell reconoce:

3. Derecho a poder recabar información sobre el contenido y composición de los procesos de selección y poder verificar, en casos concretos, la falta de personas candidatas del otro sexo" (...) 5. Ser informada clara y transparentemente sobre la estructura retributiva de la plantilla. Pudiendo proponer medidas correctoras para cumplir con el principio de equidad (...) 9. Realizar evaluaciones anuales del grado de cumplimiento, consecución de objetivos y desarrollo del Plan de Igualdad, estudiando y analizando la evolución de la situación de la mujer en el Banco.

A su vez, es necesario evitar regulaciones que perpetúen viejos patrones discriminatorios, como recocer ciertos beneficios solo a las trabajadoras durante las suspensiones del contrato de trabajo unidas a la maternidad, como sucede en el Plan de igualdad del Banco Sabadell, lo que conlleva que les lleva a incurrir en contradicciones en su propio texto:

La plantilla en situación de baja por riesgo durante el embarazo y la lactancia natural, bajas por incapacidad temporal iniciadas 30 días antes del nacimiento, permiso de maternidad y permisos de lactancia, no se excluirán del colectivo susceptible de cobrar incentivos por consecución de objetivos, y se les pagará los periodos antes mencionados en base a los siguientes criterios (...)» aunque si bien es cierto que: «El Banco garantizará al personal en situación de incapacidad temporal por riesgo durante el embarazo y la lactancia natural, permiso maternal y permiso paternal, que la percepción económica anual será igual al 100\% de su retribución fija anual (o al $100 \%$ de su salario asignado anual).

Uno de los retos más difícil es acabar con la brecha salarial de género ${ }^{20}$. En relación con ello no es muy común encontrar referencias al concepto de salario por trabajo de igual valor ni mucho menos la identificación de los elementos que determinan la desigualdad retributiva (Pons, 2015: 41). Así, es curioso

19 Sobre la importancia de estas Comisiones véase Luján y Selma (2016: 82 y ss.).

20 Por lo que respecta a posibles medidas en materia de flexibilidad interna véase: Arias(2016: 147 y ss.). 
como en el Plan de igualdad de Comedores colectivos de Celis, SL, se garantizan salarios iguales para las situaciones de embarazo y lactancia, incluido el riesgo, pero no se establecen medidas específicas para la brecha salarial que se origina en el sistema de clasificación profesional. En este sentido, los Planes de igualdad deberían fijar criterios y pautas de actuación concretas para la valoración de los puestos de trabajo y de la estructura salarial, fundamentalmente de los conceptos básicos que la integran, para garantizar unos sistemas salariales neutros y libres de discriminación indirecta (Martínez, 2015: 412).

Dentro de las posible medidas a adoptar para eliminar la brecha salarial deberían adoptarse medidas que eliminen el impacto negativo que sobre los salarios tienen las interrupciones a lo largo de la vida laboral, lo que es especialmente gravoso para las mujeres, puesto que son el grupo que presenta interrupciones más frecuentes y de mayor duración al ser ellas las que más disfrutan de los permisos por maternidad y atención a los hijos ${ }^{21}$. También tendrían que establecer sistemas de clasificación profesional que tengan en cuenta estas interrupciones de cara a una cobertura de vacantes o promociones por el impacto directo que pueden tener en la estructura retributiva (Martínez, 2015: 412). El reto es especialmente complejo ya que estas medidas deberían de acompañarse de procesos de valoración de las características propias del trabajo femenino e incorporarlas al sistema de relaciones laborales en su conjunto, lo que supondrían la incorporación de la vertiente activa y no pasiva de la igualdad efectiva o real (Perán, 2014: 76). Por ejemplo, existen diferencias relevantes por sexos en cuanto al disfrute de la jornada continua y partida, el trabajo nocturno o el trabajo en fin de semana o festivos que afectan directamente a la retribución y sobre las cuales los Planes de igualdad podrían actuar (CES, 2016: 71 y ss.).

Otro aspecto fundamental es la regulación y ordenación del tiempo de trabajo por su impacto directo en la participación laboral femenina y sus condiciones laborales (Velasco, 2016: 193 y ss.). Por ello, sería necesario que en los Planes de igualdad se analizase las diferencias en la duración y la distribución del tiempo de trabajo entre hombres y mujeres en cada empresa. En este sentido, las propuestas de actuación más recientes giran en torno al establecimiento de la

21 CES (2016: 68 y ss.) menciona que: «Es significativo, a este respecto, el menor peso relativo de los complementos salariales en el salario de las mujeres, precisamente aquel componente salarial que muestra mayores diferencias por género y que apunta a la presencia de factores determinantes de la brecha salarial como la disponibilidad horaria, la prolongación de jornada o la antigüedad en la empresa. Tales aspectos se utilizan frecuentemente para la definición de complementos del salario, y pertenecen al terreno de la configuración de la estructura salarial en las empresas, una materia típica de la negociación colectiva. En definitiva, unas trayectorias laborales más segmentadas como consecuencia de interrupciones más frecuentes ligadas a esas necesidades, y, en buena medida, las percepciones y las expectativas sobre el papel de las mujeres en la asunción de las responsabilidades familiares, alimentan la persistencia de las diferencias retributivas, a partir de una menor participación de las mujeres en los componentes salariales que priman los aspectos mencionados". 
jornada semanal efectiva ${ }^{22}$ y de medidas que fomente el ejercicio flexible del derecho, potenciando las posibilidades del artículo 38.4 ET para la adaptación de la jornada con fines de conciliación y de corresponsabilidad entre trabajadores y trabajadoras (Martínez, 2015: 413) ${ }^{23}$.

\section{A modo de conclusión}

El reto actual de los Planes de igualdad es que no se conviertan en un elenco de buenas prácticas, sino de medidas efectivas y eficaces para la erradicación de la discriminación laboral de la mujer, consecuencia de su desigual posición en la sociedad. Para lograrlo, es fundamental que partan de la realidad socio laboral de su ámbito de actuación para establecer acciones concretas, que cuenten con una coherencia interna en todo su contenido, evitando compartimentos estancos e inconexos entre sí según las áreas de actuación. Por ello es tan importante el carácter transversal y homogéneo de los mismos.

Evidentemente, hay actuaciones que únicamente corresponden a los poderes públicos, como las políticas activas de empleo, incentivos al empleo o formación, apoyo al autoempleo y el emprendimiento o las políticas públicas de apoyo a la conciliación de la vida laboral y familiar, incluidas las prestaciones de la Seguridad Social, pero también es cierto que son los que marcan las pautas de actuación para los Planes de igualdad. En relación con este aspecto, sería oportuno que la legislación abordase la definición del concepto de sexo menos representado o subrepresentado, por su impacto en esta materia, ya que legitima no solo la adopción de medidas de acción positiva, sino que también tiene otras connotaciones laborales, en concreto con la segregación laboral horizontal y vertical que tan unido está a la brecha salarial ${ }^{24}$. A su vez, este concepto debería in-

22 CES (2016: 69) señala que: «los hombres dedican más horas al trabajo remunerado, alcanzando sus jornadas semanales 48 horas o más (especialmente los autónomos), que las mujeres (menos de 20 horas). Las mujeres, sin embargo, trabajan más horas en general (si se incluyen el trabajo remunerado principal o secundario, el tiempo de desplazamiento y el trabajo no remunerado)".

${ }^{23}$ Frente a ello, existen medidas como la distribución irregular de la jornada de trabajo, reduciendo las posibilidades de organización de horarios y de compatibilidad entre tiempo de trabajo y el tiempo de vida de los trabajadores.

${ }^{24}$ Respecto a esta cuestión el principal problema que se plantea es la referencia al sexo menos representado en el ámbito de la igualdad entre sexos en la relación laboral, concepto al que no hace referencia la noción de medida de acción positiva del Real Decreto Legislativo 1/2013, de 29 de noviembre, $\mathrm{BOE}$ n. ${ }^{\circ} 289$, por el que se aprueba el Texto Refundido de la Ley General de derechos de las personas con discapacidad y de su inclusión social, en su apartado 2.g): «Medidas de acción positiva: son aquellas de carácter específico consistentes en evitar o compensar las desventajas derivadas de la discapacidad y destinadas a acelerar o lograr la igualdad de hecho de las personas con discapacidad y su participación plena en los ámbitos de la vida política, económica, social, educativa, laboral y cultural, atendiendo a los diferentes tipos y grados de discapacidad». 
cluir en su conceptualización situaciones propias del trabajo femenino y no delimitarse como un concepto neutro desde el punto de vista del sexo, como son las paradas de larga duración, a la hora de la contratación, o bien mujeres que quieren incorporarse o reincorporarse al mercado laboral tras ausencias del mismo por atención y cuidado de hijos, por medio de preferencias de acciones formativas en sectores que con demanda de empleo, a poder ser no feminizados para evitar la brecha salarial por género y en los que no exista una sobrecualificación del trabajo femenino.

\section{Bibliografía}

ARÍAs Domínguez, Ángel (2016): «Flexibilidad interna: movilidad funcional y geográfica» en SÁnchez Trigueros, Carmen (Dir.): El principio de igualdad en la negociación colectiva, Madrid, Ministerio de Empleo y Seguridad Social.

Cabeza Pereiro, Jaime (2012): «La discriminación retributiva pro razón de sexo como paradigma de discriminación sistemática», Lan Harremanak, n. 25.

Consejo EConómico y Social (CES) (2016): Informe 5/2016. La participación laboral de las mujeres en España. http://www.ces.es/documents/10180/3557409/Inf0516.pdf, Madrid.

EUROFOUND (2016): 6th European Working Conditions Survey, Luxembourg. https:// www.eurofound.europa.eu/es/publications/report/2016/working-conditions/sixtheuropean-working-conditions-survey-overview-report.

- (2017) Occupational change and wage inequality: European Jobs Monitor. Luxembourg,2017.https://www.eurofound.europa.eu/publications/report/2017/occupational-change-and-wage-inequality-european-jobs-monitor-2017.

Figueroa Domeco, Cristina y Segovia Pérez, Mónica (Coord.) (2016): Brecha salarial e igualdad de género en las empresas turístico-hoteleras, Madrid, Editorial Fundamentos.

GaRRIGUes GimÉNeZ, Amparo (Dir.) (2017): Clasificación profesional y discriminación por razón de sexo en la negociación colectiva. Un análisis multi e intersectorial, Madrid, Editorial Reus.

López GómeZ, José Manuel (2015): «Negociación colectiva, régimen económico y segmentación laboral» en CRUZ VILLALón, Jesús (Coord.): El impacto de la negociación colectiva sobre la segmentación laboral: Causas determinantes e instrumento de contención, Valencia, Tirant lo Blanch.

Luján Alcaraz, José y Selma Penalva, Alejandra (2016): «Planes de igualdad» en SánChez Trigueros, Carmen (Dir.): El principio de igualdad en la negociación colectiva, Madrid, Ministerio de Empleo y Seguridad Social.

- (2016): «Las Comisiones de igualdad» en Sánchez Trigueros, Carmen (Dir.): El principio de igualdad en la negociación colectiva. Madrid, Ministerio de Empleo y Seguridad Social.

Mercader Uguina, Jesús y Pérez Del Prado, Daniel (2010): «Ley de igualdad, negociación colectiva y planes de igualdad: balance de resultados» en BENGOECHEA GIL, María Ángeles: La lucha por la igualdad efectiva de mujeres y hombres. Reflexiones y aportaciones de la Ley de Igualdad 3/2007, de 22 de marzo. Madrid, Dykinson. 
Martínez Moreno, Carolina (2015): «Últimas tendencias en los contenidos de la negociación colectiva en materia de igualdad entre mujeres y hombres» en PERrote EsCartín, Ignacio y Mercader Uguina, Jesús (Dir.): Cláusulas de vanguardia y problemas prácticos de la negociación colectiva actual, Valladolid, Lex Nova, Thomson Reuters.

Ministerio de Sanidad, Servicios Sociales e Igualdad (2017): Guía de buenas prácticas "Medidas más eficaces para la igualdad de oportunidades entre mujeres y hombres", Colección EME, Madrid, http://www.igualdadenlaempresa.es/recursos/monograficos/ docs/BBPP_mejores_medidas_para_igualdad.pdf

Nieto Rojas, Patricia (2015): «Los planes e igualdad entre la Ley y la negociación colectiva: un diálogo insuficiente», Revista de Información Laboral, n. ${ }^{\circ} 10$.

Perán Quesada, Santiago (2014): Derecho social y género. El camino a la igualdad efectiva en las relaciones sociolaborales, Pamplona, Thomson Reuters, Aranzadi.

Pons Parera, Eva (2015): «Igualdad retributiva y garantías contra la discriminación por razón de sexo: una aproximación constitucional» en AA.VV.: Un futuro sin brecha, Albacete, Bomarzo.

Olarte Encabo, Sofía (2016): «Los planes de igualdad: los parientes pobres de la negociación colectiva» en Moya Amador, Rosa (Dir.) y Serrano Falcón, Carolina (Coord.): Estudios sobre diversos aspectos jurídicos del trabajo de la mujer, Pamplona, Thomson Reuters, Aranzadi. Organización Internacional del Trabajo (OIT): Perspectivas Sociales y del Empleo en el Mundo: Tendencias del empleo femenino 2017 http://www.ilo.org/global/research/global-reports/weso/trends-for-women2017/lang-es/index.htm.

Romero Rodenas, María Josefa (2017): Planes de Igualdad. Guía Práctica, Albacete, Bomarzo.

Servicio Público Estatal, SEPE (2017): Informe del mercado de trabajo de las mujeres estatal. Datos 2016, Madrid,, http://www.sepe.es/contenidos/que_es_el_sepe/publicaciones/pdf/pdf_mercado_trabajo/imt2017_datos2016_estatal_mujeres.pdf.

Sierra Hernáiz, Elisa (2011): «La regulación del principio de igualdad de oportunidades en la Ley Orgánica de Igualdad como límite al poder de organización y dirección de la empresa» en Sierra Hernárz, Elisa (Dir.). Eficiencia, igualdad y empresa. La aplicabilidad real a la empresa de la Ley orgánica de igualdad, Madrid, Civitas.

Velasco Portero, María Teresa (2016): «El tiempo de trabajo y su organización» en Sánchez Trigueros, Carmen (dir). El principio de igualdad en la negociación colectiva, Madrid, Ministerio de Empleo y Seguridad Social.

Vicente Palacio, Aránzazu. (2012): «En busca de la igualdad material de las mujeres: igualdad formal y medidas de acción positiva en materia de seguridad social», Lan Harremanak, núm. 25.

World ECONOMic Forum (2016): The global gender gap report 2016, http://reports. weforum.org/global-gender-gap-report-2016/ 